\section{Shape-Controlled Synthesis and Surface Plasmonic Properties of Metallic Nanostructures}

\section{Younan Xia and Naomi J. Halas, Guest Editors}

\begin{abstract}
The interaction of light with free electrons in a gold or silver nanostructure can give rise to collective excitations commonly known as surface plasmons. Plasmons provide a powerful means of confining light to metal/dielectric interfaces, which in turn can generate intense local electromagnetic fields and significantly amplify the signal derived from analytical techniques that rely on light, such as Raman scattering. With plasmons, photonic signals can be manipulated on the nanoscale, enabling integration with electronics (which is now moving into the nano regime). However, to benefit from their interesting plasmonic properties, metal structures of controlled shape (and size) must be fabricated on the nanoscale. This issue of MRS Bulletin examines how gold and silver nanostructures can be prepared with controllable shapes to tailor their surface plasmon resonances and highlights some of the unique applications that result, including enhancement of electromagnetic fields, optical imaging, light transmission, colorimetric sensing, and nanoscale waveguiding.
\end{abstract}

Keywords: field enhancement, gold nanoparticles, metallic nanostructures, shape-controlled synthesis, silver nanoparticles, surface plasmons.

\section{Introduction}

Nanostructures-structures with at least one dimension between $1 \mathrm{~nm}$ and $100 \mathrm{~nm}-$ have attracted steadily growing interest due to fascinating properties and intriguing applications that are complementary or superior to those of bulk materials. ${ }^{1}$ Much of this interest is powered by the growing expertise in fabrication methods that allow more and more ways of realizing nanostructures with well-controlled composition, size, and shape. The development of synthetic methodologies has also advanced to a level where nanostructures can be produced from many kinds of materials with the quality, quantity, and yield required for the systematic investigation of their peculiar properties (see Figure 1). The fundamental study of phenomena that occur in nanostructured materials has already evolved into a new field of research that is often referred to as nanoscience. In addition to their indispensable roles in nanoscience, nanostructures are central to the development of a broad range of emerging and exciting applications such as more powerful computer chips and higher-density information storage. It is anticipated that nanotechnology will change the way we live, just as microtechnology has done in the last century.

This issue of MRS Bulletin is focused on the shape-controlled synthesis and surface plasmonic properties of nanostructures made of two metals: gold and silver. Since the pioneering work by Gustav Mie in $1908,{ }^{2}$ it has been recognized for almost a century that the interaction of light with free electrons in a gold or silver nanoparticle can give rise to collective oscillations commonly known as surface plasmons (SPs). Peaks appear in the extinction spectra (extinction $=$ scattering + absorption) whenever SPs are excited by the electric field of incident light under the resonance condition. Such extinction peaks are responsible for the brilliant red color displayed in medieval stained glass windows, which arises from the presence of gold nanoparticles in the glass. The attractions of SPs lie in their potential to confine light to the metal/dielectric interface, which in turn generates intense local electromagnetic fields and greatly amplifies the weak signal typical of Raman scattering or second-harmonic generation. The spatial confinement of light to a structure of subwavelength dimensions also makes it possible to circumvent the optical diffraction limit, which arises because the resolution of an optical lens equals the wavelength divided by the numerical aperture, enabling the realization of nanoscale photonics.

\section{Surface Plasmons}

Plasmonic behavior is a physical concept that describes the collective oscillation of conduction electrons in a metal (or a doped semiconductor). ${ }^{3}$ Many metals (e.g., alkali metals, $\mathrm{Mg}, \mathrm{Al}$, and to some extent noble metals such as $\mathrm{Au}$ and $\mathrm{Ag}$ ) can be treated as free-electron systems whose electronic and optical properties are determined by the conduction electrons alone. In the Drude-Lorentz model, such a metal is denoted as a plasma, because it contains equal numbers of positive ions (fixed in position) and conduction electrons (free and highly mobile). Under the irradiation of an electromagnetic wave, the free electrons are driven by the electric field to coherently oscillate at a plasma frequency of $\omega_{p}$ relative to the lattice of positive ions. For a bulk metal with infinite sizes in all three dimensions, $\omega_{\mathrm{p}}$ can be expressed as

$$
\omega_{\mathrm{p}}=\left(N e^{2} / \varepsilon_{0} m_{\mathrm{e}}\right)^{1 / 2},
$$

where $N$ is the number density of electrons, $\varepsilon_{0}$ is the dielectric constant of a vacuum, and $e$ and $m_{\mathrm{e}}$ are the charge and effective mass of an electron, respectively. Quantized plasma oscillations are called plasmons.

In reality, we have to deal with metallic structures of finite dimensions that are surrounded by materials with different dielectric properties. Since an electromagnetic wave impinging on a metal surface only has a certain penetration depth $(<50 \mathrm{~nm}$ for $\mathrm{Ag}$ and $\mathrm{Au}$ ), just the electrons on the surface are the most significant. Their 


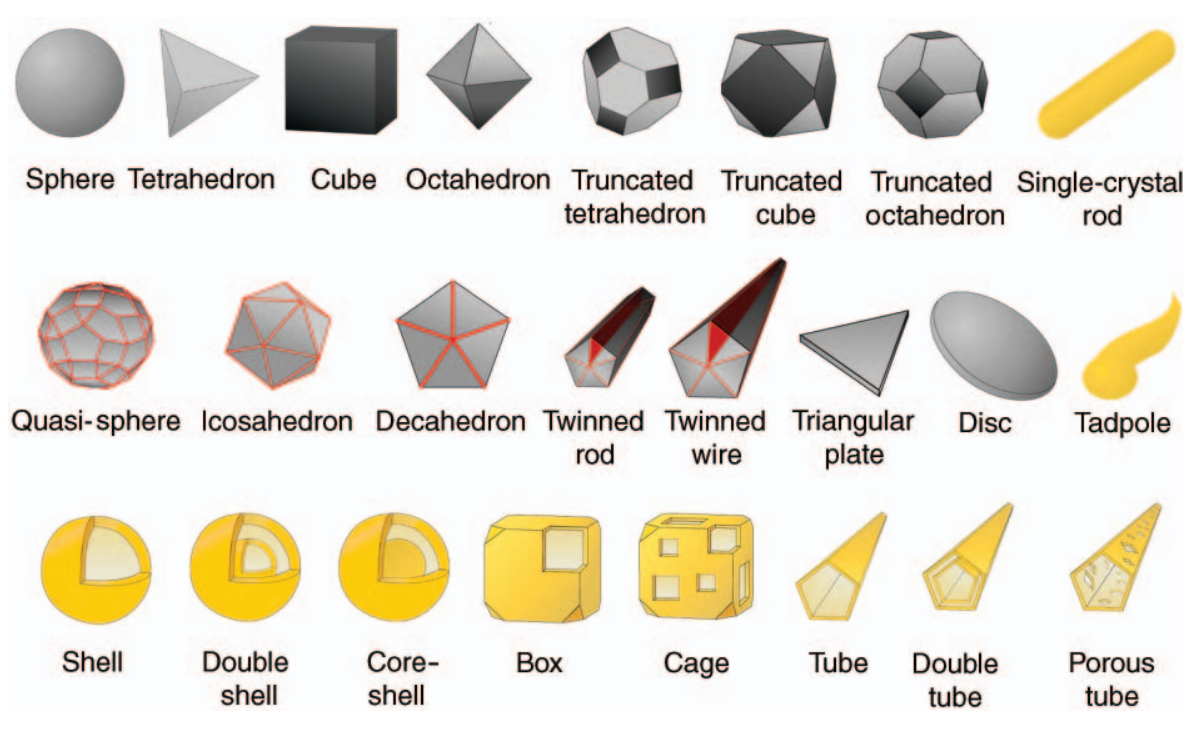

Figure 1. Schematic illustration of nanostructure shapes. The shapes in the top row are single crystals, in the second row are particles with twin defects or stacking faults, and in the third row are gold shells. All twinned and single-crystal shapes shown, with the exception of the octahedron, can be synthesized in solution. Control of shape allows control of optical and catalytic properties, as well as suitability for electronic applications in the case of wires, tubes, and possibly rods. Dark facets are (100) planes, light gray are (111) planes, and $\{111\}$ twin planes are shown in red. Gold shapes represent gold particles, and gray shapes represent silver particles, although spheres, twinned rods, icosahedrons, and cubes can also be made from gold.

collective oscillations are properly termed surface plasmon polaritons (SPPs), but are often referred to as surface plasmons (SPs). ${ }^{3-6}$ For a metal-vacuum interface, application of the boundary condition results in an SP mode of $\omega_{\mathrm{p}} / \sqrt{2}$ in frequency. As depicted in Figure 2a, such an SP mode represents a longitudinal surface charge density wave that can travel across the surface. For this reason, these SPs are also widely known as propagating SPs (or PSPs), which can be excited through a resonant mechanism by passing an electron through a thin metallic film or by reflecting an electron or a photon from the surface of a metallic film. By resonance, we mean a condition in which the frequencies and wave vectors of both incident and SP waves are approximately the same, leading to constructive interference and a stronger signal.

Another type of SP (localized SPs, or LSPs) corresponds to collective excitations of free electrons confined to a finite volume. A typical example is shown in Figure 2b, where the conduction electrons of a spherical gold colloid oscillate coherently in response to the electric field of incident light. ${ }^{4-6}$ When this process occurs in a vacuum, the resonant frequency becomes $\omega_{\mathrm{p}} / \sqrt{3}$.

For nearly a century, it has been known that the number, location, and intensity of SP bands of a gold or silver nanoparticle are strongly correlated with both the shape and size of the particle. The gold and silver systems are unique because their densities of free electrons are in the proper range to give their nanoparticles SP peaks in the visible regime. For spherical gold and silver particles of relatively small size $(<<\lambda$, the wavelength of light, with diameters in the range of $1-20 \mathrm{~nm}$ ), only dipole plasmon resonance is involved; their suspensions display a strong SP peak around $510 \mathrm{~nm}$ and $400 \mathrm{~nm}$, respectively.

\section{Fabrication of Nanostructures}

Many techniques are available for fabricating metallic nanostructures on solid supports. ${ }^{7}$ The best-established, most versatile tool is probably electron-beam lithography (EBL), which has the capability of extremely high resolution as a result of the short wavelengths associated with high-energy electrons (e.g., $\sim 0.005 \mathrm{~nm}$ for an accelerating voltage of $50 \mathrm{kV}) .{ }^{8}$ EBL is also a feature that can be readily added onto most conventional scanning electron microscopy (SEM) systems.

In typical e-beam writing, a tightly focused beam of electrons is scanned across the surface of a layer of resist such as poly(methyl methacrylate). The interaction of electrons with the resist causes local changes to the polymer chains, making the material more or less soluble in a developing solution. When combined with masked evaporation and liftoff, relief patterns in the resist film can be readily transformed into patterned metallic nanostructures with well-controlled dimensions and separations.

In practice, the resolution of e-beam writing is mainly determined by the scattering of both primary and secondary electrons in the resist film and the substrate. Structures with lateral dimensions as small as $\sim 30 \mathrm{~nm}$ can be routinely generated using EBL. Features as fine as $\sim 2 \mathrm{~nm}$ have also been demonstrated, ${ }^{8}$ using thin membranes as substrates to reduce the scattering of electrons.

A related technique, focused ion-beam (FIB) lithography, provides another attractive route to metallic nanostructures. ${ }^{9}$ It has been shown that structures as small as $\sim 6 \mathrm{~nm}$ can be fabricated by using a $50 \mathrm{kV} \mathrm{Ga}^{+}$two-lens system, which uses magnetic lenses like those in electron microscopes to focus a $\mathrm{Ga}^{+}$ion beam. ${ }^{9}$ As an advantage over EBL, this technique can be used to directly deposit metals as patterned nanostructures by decomposing suitable precursors. However, this technique is less popular in the scientific community because it requires an expensive, more dedicated instrument system. Although attaining high-throughput fabrica-
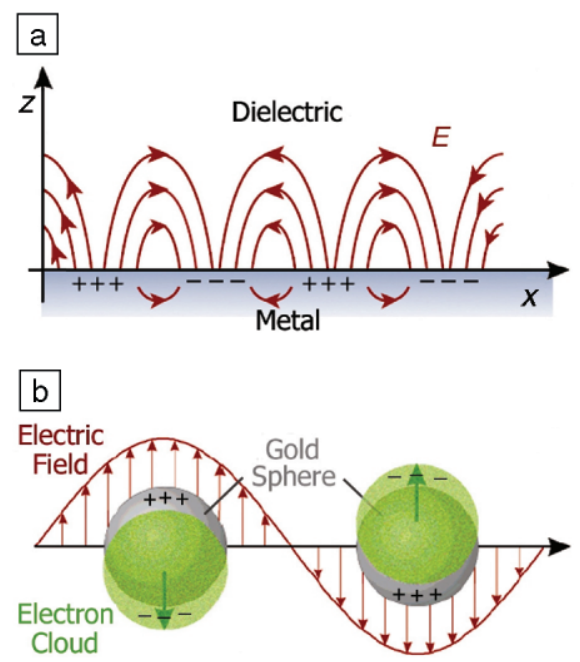

Figure 2. Schematic illustration of the collective oscillations of free electrons for (a) a metal-dielectric interface and (b) a spherical gold colloid. Excited by the electric field of incident light, the free electrons can be collectively displaced from the lattice of positive ions (consisting of nuclei and core electrons). While the plasmon shown in (a) can propagate across the surface as a charge density wave, the plasmon depicted in (b) is localized to each particle. (Courtesy of R. Van Duyne and T. Schatz,

Northwestern University.) 
tion continues to be a major challenge with these two techniques, they have become the tools of choice for producing arbitrary patterns for many technological and scientific applications.

A variety of unconventional approaches have also been demonstrated for low-cost fabrication of metallic nanostructures. One example is nanosphere lithography (NSL), illustrated in the article by Haes et al. in this issue. This technique relies on monodisperse spherical colloids that readily assemble on a flat surface to form a close-packed monolayer with hexagonal symmetry. The monolayer can serve as a physical mask in the subsequent step. For most demonstrations, a metal is evaporated through the monolayer to fill the void spaces among adjacent colloids in the monolayer lattice. Upon liftoff of the colloids, a periodic array of nanometer-sized, pyramidal islands of the metal is left behind on the substrate. The lateral dimensions of these islands and the separation between them are determined by the size of the spherical colloids, while the vertical dimension mainly depends on the amount of deposited material. When a double layer of spherical colloids is used, hexagonal dots corresponding to the small openings that remain in the close-packed lattice are formed. ${ }^{10}$ Most recently, a monolayer of spherical colloids was also used to direct the spreading of alkanethiols on a gold substrate to generate nanoscale rings of gold. ${ }^{11}$

Although NSL can be used to pattern large areas in parallel, this technique remains sensitive to defects, distortions, and domain boundaries that naturally appear when spherical colloids assemble on flat substrates. The structures and patterns that can be generated using this approach are limited in terms of design and complexity. In this regard, unconventional approaches based on soft lithography ${ }^{12}$ and dip-pen nanolithography (DPN) ${ }^{13}$ might provide some immediate advantages.

\section{Chemical Synthesis of Gold and Silver Nanostructures}

The first documented synthesis of metallic nanostructures can be traced back to 1856 when Michael Faraday demonstrated the preparation of gold colloids by reducing an aqueous solution of gold chloride with phosphorus. His samples displayed a stable, ruby-red color, and some of them are still preserved today in the Faraday Museum in London. ${ }^{14}$

In the past 150 years, a wealth of methodologies has been developed for processing metals such as gold and silver into nanoscale structures. However, most of the products are characterized by problems such as irregular shapes, polydispersed sizes, and poorly defined structures. Only within the last decade have chemical methods been established for generating high-quality gold and silver nanostructures in quantity, with the reproducibility required for a systematic study of the dependence of surface plasmonic resonance (SPR) properties on size, shape, and structure. ${ }^{15}$ Some of these developments are briefly covered in the articles by Murphy et al., Wiley et al., and Halas in this issue.

Murphy and co-workers review the chemical synthesis of one-dimensional nanostructures of gold and silver via surfactant-directed routes. In a typical process, a precursor such as $\mathrm{HAuCl}_{4}$ or $\mathrm{AgNO}_{3}$ is reduced to atomic species by chemical, electrochemical, or photochemical means in the presence of ionic surfactants at high concentrations. The aspect ratios (ratio of length to width) of resultant nanostructures can be easily varied over a wide range (up to 23) by adjusting the experimental parameters.

These authors also discuss the surface plasmonic properties associated with gold or silver nanorods. ${ }^{16}$ When a spherical particle is elongated, the SP band is split into two: the transverse band (along the short axis) and longitudinal band (along the long axis). While the transverse band is located at roughly the same position as that of the spherical particle, the longitudinal band can be continuously shifted into the nearinfrared region by increasing the aspect ratio. Such control allows one to fine-tune the resonance wavelength at which the electromagnetic field will be enhanced.

Wiley et al. discuss a different approach, based on polyol reduction, to the large-scale synthesis of silver nanostructures with a variety of well-defined shapes. The seeds formed in the nucleation step are either twinned or single-crystalline, depending on the absence or presence of an oxidative etchant. In the presence of a suitable capping agent, the seeds can be directed to grow into nanostructures in the form of cubes, wires, quasi-spheres, or triangular plates. These authors also describe the use of a galvanic replacement reaction between silver nanostructures and $\mathrm{HAuCl}_{4}$ in an aqueous medium as a powerful route to making gold nanocages-colloidal particles having hollow interiors and porous walls. It is worth noting that the SPR peaks of gold nanocages can be continuously shifted from blue $(425 \mathrm{~nm})$ to nearinfrared (1200 $\mathrm{nm})$ by simply adjusting the molar ratio between $\mathrm{Ag}$ and $\mathrm{HAuCl}_{4}$ (in a fashion similar to titration).

Halas discusses the synthesis and unique SP properties of gold/silver nanoshellsconcentric nanoparticles consisting of dielectric cores and metallic shells. In contrast to gold or silver solid colloids, the SP bands of such core-shell nanoparticles can be continuously shifted from the visible to the near-infrared region (where blood and soft tissues are optically transparent) by controlling the diameter or wall thickness of the nanoshells. Such a tuning capability has enabled a variety of biomedical applications, including optical sensing, imaging, and drug delivery. The author also discusses a model-plasmon hybridization-that her group has recently proposed to understand the optical resonant properties of gold nanoshells. In this new model, the coupling of plasmons follows a mesoscale analogue of molecular orbital theory, hybridizing in precisely the same manner as the individual atomic wave functions in simple molecules. This model can be used to effectively predict SP properties of metallic nanostructures and is expected to play an important role in the design and synthesis of structures with specific plasmonic features.

\section{Light-Scattering and Absorption}

Understanding the extinction spectra of gold and silver nanoparticles has long been of interest to the scientific community, dating back at least to Faraday's investigations of gold colloids in the middle of the 19th century. In 1908, Gustav Mie solved Maxwell's equations and presented the exact formula for calculating both scattering and absorption cross sections of a spherical particle of arbitrary size. ${ }^{2}$ Mie's solution remains of great interest and value to this day, although it can only be applied to spherical particles. In the past decade, a number of numerical methods have been developed to calculate the optical properties of small particles of arbitrary shapes; a notable example includes the discrete dipole approximation (DDA) method.

Thanks to the efforts of several groups, the DDA method has emerged as a powerful tool for calculating the scattering/ absorption cross sections of small particles. ${ }^{17}$ In this method, the particle is approximated as an array of polarizable cubic elements, with the array being large enough for the calculation to converge. The scattering and absorption cross sections of the particle can be obtained once the location and polarizability of each element have been specified. Figures $3 \mathrm{a}$ and $3 \mathrm{~b}$ show the spectra calculated using this method for spherical gold colloids of $20 \mathrm{~nm}$ and $50 \mathrm{~nm}$ in radius, respectively. In general, light absorption dominates the extinction spectrum for particles of relatively small radius $(<20 \mathrm{~nm})$, and light-scattering becomes the dominant process for large particles. As the particles increase in size, the SP peaks are usually shifted toward the 


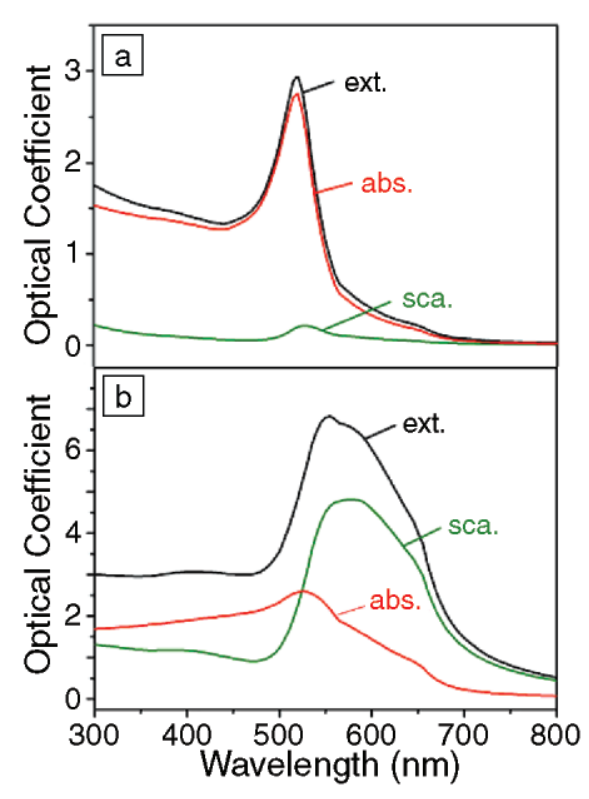

Figure 3. Optical coefficients calculated using the discrete dipole approximation method for a spherical gold colloid with (a) radius $r=20 \mathrm{~nm}$ and (b) $r=50 \mathrm{~nm}$ (ext. stands for extinction, abs. for absorption, and sca. for scattering). Water was taken as the surrounding medium. In plotting the spectra, the calculated cross sections were divided by $\pi r^{2}$ to obtain the dimensionless optical coefficients (Q). The calculations were performed by Z.-Y. Li at the Institute of Physics, Chinese Academy of Sciences.

red wavelengths. New peaks may also appear in the extinction spectra due to the excitation of quadrupole modes.

When compared with molecular species such as organic chromophores, the absorption and scattering cross sections of gold and silver nanoparticles are 5-6 orders in magnitude higher. As a result, these nanoparticles have recently been explored as a class of therapeutic and contrast agents for photothermal treatment and optical imaging of tumors, respectively. ${ }^{18,19}$ The use of gold and silver makes it straightforward to derivatize their surfaces with various functional groups by taking advantage of the well-established monolayer chemistry based on alkanethiols. It has also become increasingly apparent that one can precisely control the wavelengths at which gold and silver nanoparticles absorb and scatter light by controlling their shapes, dimensions, and structures (e.g., solid versus hollow). In addition, it is possible to tailor the magnitude of absorption and scattering coefficients by engineering their geometric parameters. All of these advances will greatly boost the use of gold and silver nanostructures in biomedical applications such as optical imaging and the treatment of cancer.

\section{Colorimetric Sensing}

The sensitive response of SP peaks to environmental changes can be exploited to optically detect and monitor binding events on the gold or silver surface. The most popular detection scheme is based on the excitation of propagating SPs in a metallic thin film deposited on a transparent substrate. ${ }^{20}$ Commercial instruments are available from a number of companies such as Biacore and Texas Instruments.

In addition to thin films, the localized SPs of gold or silver colloids have been widely explored for optical sensing in two different configurations. The first configuration involves the use of individual nanoparticles and is discussed by Haes et al. in this issue. According to the scattering theory, any variation in the refractive index of the surface layer will lead to some changes in the intensity and/or position for the LSP peak, and an increase in the refractive index often causes the LSP peak to shift to the red.

Based on this mechanism, gold nanoparticles supported on substrates or suspended in solutions have been used by several groups to demonstrate label-free optical sensing. ${ }^{21}$ The sensitivity of such an optical probe is strongly dependent on the size, shape, composition, and structure of the nanoparticles. For example, the sensitivity factor (i.e., the shift in peak position per unit change in the refractive index of the surrounding medium) was reported to be $76.4 \mathrm{~nm}$ per refractive index unit (RIU), defined as a change of 1 in the refractive index, and $191 \mathrm{~nm} / \mathrm{RIU}$, respectively, for a submonolayer of gold solid colloids (diameter, $13.4 \mathrm{~nm}$ ) and a hexagonal array of silver islands (in-plane width, $100 \mathrm{~nm}$; out-of-plane height, $50 \mathrm{~nm}$ ) fabricated using NSL. The sensitivity factor could be increased to $408.8 \mathrm{~nm} / \mathrm{RIU}$ for gold nanoshells of $50 \mathrm{~nm}$ in diameter and $4.5 \mathrm{~nm}$ in wall thickness. ${ }^{22}$ In general, the spectral shift is relatively insignificant $(<20 \mathrm{~nm}$ in wavelength), and a spectrometer is needed to detect the change in peak position.

Thaxton et al. discuss the second detection configuration, which relies on the redshift of the SP peak accompanying the aggregation of gold or silver particles due to the coupling between their LSP modes. This configuration is distinguished from the first detection configuration by the presence of an analyte that can be visually detected by the naked eye, as long as the analyte molecules can selectively induce the colloids to aggregate into larger particles. This approach has been employed by the Mirkin group to qualitatively detect DNA and single-base mismatches in DNA hybridization, and by the Rosenzweig group for immunoassays to probe the presence of proteins. ${ }^{23}$

Aggregation-induced color changes due to the formation of sandwich complexes have also been explored by the Hupp group to measure the concentration of metal ions such as $\mathrm{K}^{+}, \mathrm{Pb}^{2+}, \mathrm{Cd}^{2+}$, and $\mathrm{Hg}^{2+}$ through an ion-templated chelating process. ${ }^{24}$ Because the surface of gold and silver nanoparticles can be readily bioconjugated to a number of binding groups, including oligonucleotides, proteins, and other biologically relevant ligands, it is feasible to design and synthesize optically and chemically encoded nanoparticle probes that can support biomolecular assays with numerous advantages over conventional methods, such as low cost and high sensitivity. It is believed that bioconjugated gold and silver nanoparticles will become the centerpiece for a variety of detection strategies.

\section{Enhancement of Light Transmission}

When light is transmitted through an aperture with lateral dimensions much smaller than the wavelength of the light, the efficiency is extremely low $\left(\sim 10^{-3}\right.$ for a 150-nm-diameter hole), because the photons have to use an inefficient mechanism to tunnel through.

This picture may change completely if the hole is fabricated in a thin film of silver or gold. In this case, propagating surface plasmons can be activated in the metallic film to substantially enhance the transmittance of light at resonance wavelengths. ${ }^{29}$ Dintinger et al. review the current understanding of this phenomenon and illustrate how it can be applied to greatly improve the performance of various types of photonic devices.

In a typical experiment, an aperture (hole or slit) is fabricated in a silver or gold thin film suspended or supported on a transparent substrate, and transmission spectra are recorded. It is critical to have the aperture surrounded by a periodic corrugation in order to facilitate coupling between the incident light and the SP modes.

For a periodic array of holes fabricated in a suspended film, the light couples to the SP modes at the input interface and exponentially decays across the film. This propagation causes an energy transfer toward the output interface where SP modes are excited and then decoupled into freely propagating light. The transmission spectra display peaks at wavelengths where the SP modes are excited. 
The transmittance normalized to the aperture area can be orders of magnitude greater than that predicted by classic diffraction theory. For systems with asymmetric interfaces (e.g., a silver film supported on a quartz substrate), the SP modes at the input and output sides are different in frequency. As a result, the transmission spectrum contains two series of maxima associated with each interface that are offset by the difference in refractive index for the media in contact with the metal surfaces. In addition to the enhancement of transmission, it is feasible to concentrate the transmitted light into a narrow beam with an angular divergence of less than a few degrees by surrounding the aperture with an appropriate grating on the exit side of the metallic film. These demonstrations illustrate how surface plasmons can be exploited to control and manipulate light at the subwavelength scale.

\section{Nanoscale Waveguiding}

It has been demonstrated both theoretically and experimentally that a linear chain of gold or silver nanoparticles can channel the flow of electromagnetic energy over distances of hundreds of nanometers without significant loss. ${ }^{25}$ The major requirement is that these nanoparticles are separated by gaps narrow enough $(<1 \mathrm{~nm})$ to enable near-field coupling between the SPR modes associated with individual nanoparticles.

In general, there is a tradeoff between energy loss and spatial localization in plasmonic waveguides, but for important visible and near-infrared wavelengths, it should be feasible to achieve centimeterscale propagation. In practice, the metallic plasmonic structures can serve as interconnects and can be potentially adopted to create chip-scale integrated photonics and nanoscale all-optical networks. It is anticipated that SPR coupling will become as important as $p-n$ junctions, enabling the realization of nanoscale photonics, where optical devices can be scaled down to dimensions of less than $10 \%$ of the free space wavelength.

Atwater and co-workers describe the fabrication of nanoscale optical waveguides by taking advantage of the SPR coupling between adjacent metallic nanostructures. In such waveguiding structures, the electromagnetic wave can transport with a spatial confinement well below the diffraction limit $(<100 \mathrm{~nm})$. A number of methods have been explored for fabricating plasmonic waveguides, including e-beam lithography, high-energy ion irradiation, and selfassembly. Although e-beam writing can routinely generate ordered arrays of metallic nanoparticles with well-controlled dimen- sions and spatial separations, the development of this technique into a practical method for producing large numbers of such nanostructures rapidly and at low cost still requires great ingenuity. It is also nontrivial to control the gap between adjacent nanoparticles below a few nanometers.

In contrast, self-assembly provides a number of attractive features in organizing metallic nanoparticles into ordered arrays. As illustrated in the articles by Murphy et al. and Wiley et al. in this issue, both the size and shape of the building blocks (i.e., nanoparticles) can be readily altered to tailor their SPR features, and the spacing between adjacent nanoparticles can also be controlled by coating their surfaces with thin dielectric shells.

\section{Enhancement of Electromagnetic Fields}

In addition to the scattering and absorption of light, gold or silver nanostructures can be used to substantially enhance local electromagnetic fields. Upon excitation of SPs, charges will be concentrated at the metal-dielectric interface, resulting in very strong amplification of the electric field (E). For colloidal particles 10-200 nm in size where LSPs are excited, the $|\mathbf{E}|^{2}$ can be 100 to 10,000 times greater in magnitude than the incident field. The distribution of field has a spatial range on the order of $10-50 \mathrm{~nm}$ and is strongly dependent on the size, shape, and local dielectric environment of the particle. For smooth thin films with thicknesses in the range of 10-200 nm, the excitation of PSPs only leads to field enhancements $\left(|\mathbf{E}|^{2}\right)$ on the order of 10-100, although the field can extend over a longer spatial range $(\sim 1000 \mathrm{~nm})$.

The ability of gold and silver nanostructures to enhance the local electric fields has led to the development of a new field known as surface-enhanced spectroscopy. Haes et al. present an overview of some exciting developments in this area. Halas and Murphy et al. also provide some brief discussions on this subject in their articles.

A typical example can be found in surfaceenhanced Raman scattering (SERS), ${ }^{26}$ a phenomenon that was discovered almost 30 years ago. SERS is a complicated process whose operational mechanism can be attributed to at least two factors: electromagnetic enhancement and chemical enhancement. Electromagnetic enhancement occurs when the incident light is in resonance with the SP modes of a metallic thin film or nanoparticle. In this case, it is crucial to fine-tune the spectral positions of SPs to achieve the resonance condition. Gold and silver nanostructures with controllable shapes are excellent candidates for this application.
Chemical enhancement is related to the possible chemical interactions, including charge transfer and polarization, that may occur between adsorbed molecules and the surface. In addition to SERS, surfaceenhanced fluorescence has also been reported for chromophores near the surfaces of metallic nanoparticles. ${ }^{27}$ When the molecules are placed within $\sim 5 \mathrm{~nm}$ of the metal nanoparticle surface, fluorescence is quenched. However, the fluorescence can be enhanced up to 100 -fold by the localized electric field if the molecules are separated from the metal surface by $\sim 10 \mathrm{~nm}$ or more. Furthermore, the enhancement of local field has also been used to amplify the weak signals from nonlinear optical processes such as second-harmonic generation $(\mathrm{SHG}) .^{28}$ It is expected that surfaceenhanced spectroscopy will play an important role in single-molecule detection.

\section{Summary and Outlook}

Surface plasmons clearly have a broad range of technological applications. They provide a powerful tool for controlling, manipulating, and amplifying light on the subwavelength scale, enabling the realization of highly complex nanoscale devices such as sensors, light sources, filters, polarizers, and waveguides. For this technology to reach its paramount potential, active plasmonic devices such as switches and modulators still need to be demonstrated. In recent work by Andrew and Barnes, an important step forward was made in showing that SPs could be used to effectively transfer energy from donor to acceptor molecules separated by silver films up to $120 \mathrm{~nm}$ thick. ${ }^{30}$ Such a molecular plasmonic device might lead to the fabrication of more efficient light-emitting diodes and photovoltaics.

For most of the applications associated with SPs, generating metallic nanostructures with controllable sizes, shapes, and structures represents the first and one of the most significant challenges to their realization. Compared with nanofabrication techniques, chemical synthetic methods are still in a rudimentary stage of development. There are many fundamental issues that need to be solved before they can become the methods of choice for industrial applications. For example, the exact nucleation and growth mechanisms involved in most of the synthetic methods for creating metallic nanoparticles remain mysterious because of the length and time scales on which they occur. Although nuclei or seeds may play the most important role in determining the morphology of final products, it is extremely difficult to probe their structures experimentally. In fact, they might assume crystal structures different from 
the bulk solids, and their catalytic activities might strongly depend on their size and shape. All of these unknowns make it difficult to decipher the mechanism responsible for shape-controlled synthesis.

Our goal with this issue is to provide readers with some representative and exciting snapshots of the new developments occurring in the field of surface plasmons. Due to the highly dynamic nature of this fast-evolving area, it is impossible to cover every aspect of the subject. There is no doubt that research on fabrication and synthetic strategies will continue to develop strongly, with contributions coming from chemists, physicists, materials scientists, and engineers. We sincerely hope that readers will not only enjoy the topics presented here, but perhaps also find the inspiration to push this field to the next level of success.

\section{References}

1. M. Di Ventra, S. Evoy, and J.R. Heflin Jr., eds., Introduction to Nanoscale Science and Technology (Kluwer Academic Publishers, Boston, 2004).

2. G. Mie, Ann. Phys. 25 (1908) p. 377.

3. C. Kittel, Introduction to Solid State Physics, 6th ed. (John Wiley \& Sons, New York, 1986).
4. C.F. Bohren and D.R. Huffman, Absorption and Scattering of Light by Small Particles (John Wiley \& Sons, New York, 1983).

5. U. Kreibig and M. Vollmer, Optical Properties of Metal Clusters (Springer-Verlag, New York, 1995). 6. E. Hunter and J.H. Fendler, Adv. Mater. 16 (2004) p. 1685.

7. M. Geissler and Y. Xia, Adv. Mater. 16 (2004) p. 1249.

8. J.M. Gibson, Phys. Today (October 1997) p. 56 9. D. Brambley, B. Martin, and P.D. Prewett, Adv. Mater. Opt. Electron. 4 (1994) p. 55.

10. C.L. Haynes and R.P. Van Duyne, J. Phys. Chem. B 105 (2001) p. 5559.

11. J. McLellan, M. Geissler and Y. Xia, J. Am. Chem. Soc. 126 (2004) p. 10830.

12. Y. Xia and G.M. Whitesides, Angew. Chem. Int. Ed. Engl. 37 (1998) p. 550.

13. D.S. Ginger, H. Zhang, and C.A. Mirkin, Angew. Chem. Int. Ed. Engl. 43 (2004) p. 30.

14. "Heritage: Faraday page," the Royal Institution of Great Britain, http://www.rigb.org/ rimain/heritage/faradaypage.jsp (accessed March 2005).

15. B. Wiley, Y. Sun, B. Mayers, and Y. Xia, Chem. Euro. J. 11 (2005) p. 455.

16. M.A. El-Sayed, Acc. Chem. Res. 34 (2001) p. 257.

17. K.L. Kelly, E. Coronado, L.L. Zhao, and G.C. Schatz, J. Phys. Chem. B 107 (2003) p. 668.

18. L. Hirsch, R. Stafford, J. Bankson, S. Sershen, B. Rivera, R. Price, J. Hazle, N. Halas, and J. West, Proc. Natl. Acad. Sci. U.S.A. 100 (2003) p. 13549

19. J.G. Fujimoto, Nature Biotechnol. 21 (2003) p. 1361.

18. I.O. Sosa, C. Noguez, and R.G. Barrera, J. Phys. Chem. B 107 (2003) p. 6269.

20. J.M. Brockman, B.P. Nelson, and R.M. Corn, Annu. Rev. Phys. Chem. 51 (2000) p. 41.

21. N. Nath and A. Chilkoti, Anal. Chem. 74 (2002) p. 504.

22. Y. Sun and Y. Xia, Anal. Chem. 74 (2002) p. 5297.

23. N.T.K. Thanh and Z. Rosenzweig, Anal. Chem. 74 (2002) p. 1624.

24. Y. Kim, R.C. Johnson, and J.T. Hupp, Nano Lett. 1 (2001) p. 165.

25. S.A. Maier, P.G. Kik, H.A. Atwater, S. Melyzer, E. Harel, B. Koel, and A.A.G Requicha, Nature Mater. 2 (2003) p. 229.

26. K. Kneipp, H. Kneipp, I. Itzkan, R.R. Dasari, and M.S. Feld, Chem. Rev. 99 (1999) p. 2957.

27. A. Parfenov, I. Gryczynksi, J. Malicka, C.D. Geddes, and J.R. Lakowicz, J. Phys. Chem. B 107 (2003) p. 8829

28. Y. Shen, C.S. Friend, Y. Jiang, D. Jakubczyk, J. Swiatkiewicz, and P.N. Prasad, J. Phys. Chem. B 104 (2000) p. 7577.

29. W.L. Barnes, A. Dereux, and T.W. Ebbesen, Nature 424 (2003) p. 824.

30. P. Andrew and W.L. Barnes, Science 306 (2004) p. 1002.
Younan Xia, Guest Editor for this issue of MRS Bulletin, is a professor of chemistry at the University of Washington. He received his BS degree in chemical physics from the University of Science and Technology of China (USTC) in 1987 and then worked as a graduate student on inorganic nonlinear optical crystals at the Fujian Institute of Research on the Structure of Matter, Academia Sinica. He earned his MS degree in inorganic chemistry from the University of Pennsylvania (with Alan G. MacDiarmid) in 1993 and his $\mathrm{PhD}$ degree in physical chemistry from Harvard University (with George M. Whitesides) in 1996. He then stayed at Harvard and worked as a postdoctoral fellow with both Whitesides and Mara Prentiss. He began as an assistant professor of chemistry at the University of Washington in 1997.
Xia's research interests include nanostructured materials, self-assembly, photonic crystals, colloidal chemistry, microfabrication, surface modification, electrospinning, conducting polymers, microfluidics, and novel devices for photonics, optoelectronics, and displays.

He has received many awards, including a National Science Foundation CAREER Award, the Camille Dreyfus Teacher-Scholar Award, and the Victor K. LaMer Award from the American Chemical Society. He has been an Alfred P. Sloan Research Fellow and a David and Lucile Packard Fellow. Xia is the co-author of more than 200 peer-reviewed publications and has edited a number of special issues and books on nanostructured materials. He currently serves as an associate editor for Nano Letters.
Xia can be reached by e-mail at xia@chem. washington.edu.

Naomi J. Halas, Guest Editor for this issue of MRS Bulletin, is the Stanley C. Moore Professor of Electrical and Computer Engineering and a professor of chemistry at Rice University. She received her undergraduate degree in chemistry from La Salle University in Philadelphia, and her $\mathrm{MS}$ and $\mathrm{PhD}$ degrees in physics from Bryn

Mawr College, the latter while she was a graduate fellow at IBM's T.J. Watson Research Center. Following her postdoctoral research at AT\&T Bell Laboratories, she joined the faculty at Rice. She is best known for her invention of nanoshells, a new type of nanoparticle with tunable optical properties especially suited for biotechnology applications. She has been

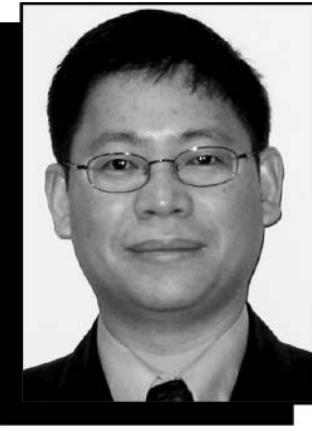

Younan Xia

honored with the NSF Young Investigator Award, three Hershel Rich Invention Awards, the 2003 Cancer Innovator Award from the Congressionally Directed Medical Research Programs of the Department of Defense, and the 2000 CRS-Cygnus Award for Outstanding Work in Drug Delivery. She was also awarded "Best Discovery of 2003" by Nanotechnology Now and was a finalist for the Small Times 2004 Nanotechnology Researcher of the Year.

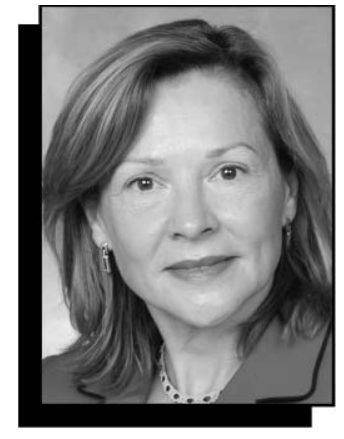

Naomi J. Halas

She has published more than 100 peer-reviewed papers, has presented over 150 invited talks, and holds nine issued patents. Halas is a fellow of the American Physical Society and of the Optical Society of America. She is also the founder and director of the Rice University Laboratory for Nanophotonics (LANP), a multidisciplinary research network whose mission is the design, invention, and application of nanoscale optical components. 


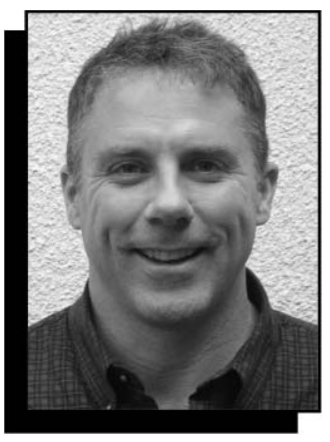

Harry A. Atwater

Halas can be reached by e-mail at halas@rice. edu.

Harry A. Atwater is the Howard Hughes Professor and a professor of applied physics and materials science at the California Institute of Technology. His research interests center on synthesis, properties, and processing of materials for use in electronic, photonic, and opto/ mechanical devices and circuits. His current research efforts are focused on plasmonic and nanocrystal optoelectronic devices; thin-film photovoltaic materials and devices, including thin-film silicon, III-V compound photovoltaic heterostructures, and nanostructure photovoltaics; and ferroelectric and piezoelectric thin-film materials.

He has co-authored over 150 refereed journal publications in the fields of electronic and phototonic materials.

Atwater received his $\mathrm{PhD}$ degree in electrical engineering from MIT in 1987. He is a recipient of the NSF Presidential Investigator Award and the AT\&T Foundation

Award and is a member of the Böhmische Physical Society and a Joop Loos Fellow of the FOM Institute, Amsterdam. He currently serves as the director of Caltech's Center for Science and Engineering of Materials (an

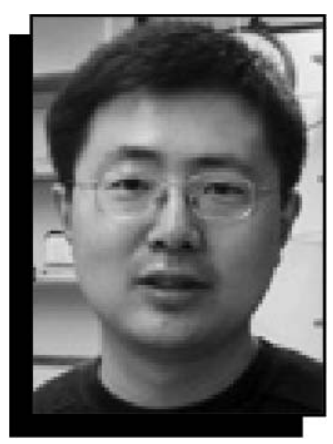

Hu Cang

NSF MRSEC) and is a member of the board of directors of the Gordon Research Conferences. $\mathrm{He}$ is founder and chief technical advisor for Aonex Corporation.

He has consulted extensively for industry and government and has also actively served the materials community as a meeting chair (1997) and as president (2000) of the Materials

Reaserch Society and as a Gordon Research Conference chair (2001).

Atwater can be reached at the California Institute of Technology, Thomas J. Watson Laboratories of Applied

Physics, Mail Code 12895, Pasadena, CA 91125, USA; tel. 626-395-2197, fax 626-844-9320, and e-mail haa@caltech. edu.

Hu Cang is a postdoctoral researcher working with Younan Xia and Xingde $\mathrm{Li}$ at the University of Washington. He received his BS degree in chemical physics from the University of Science and Technology of China (USTC) in 1998, his MS degree in electrical engineering from Stanford University in 2003, and his PhD degree in chemistry from Stanford (with Michael D. Fayer) in 2004. His research interests include nonlinear optical spectroscopy, dynamics of complex liquids, glass transitions,

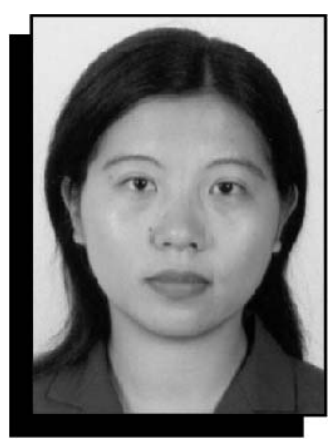

Jingyi Chen

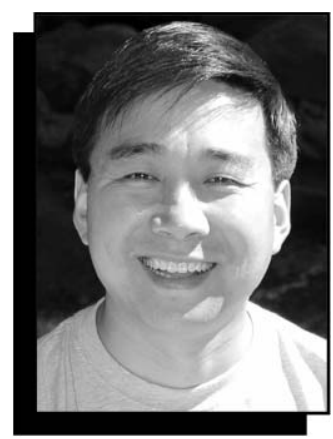

Xingde Li

and optical coherence tomography.

Cang can be reached by e-mail at hucang@ u.washington.edu.

Jingyi Chen is a graduate student in the Department of Chemistry at the University of Washington, where she works on the synthesis of nanostructured materials and functionalization of their surfaces. She received her BS degree in chemistry in 1997 from Zhong-Shan University in Guangzhou, China; her MS degree in chemistry in 2000 from the Guangzhou Institute of Chemistry, Academia Sinica; and her MS degree in biochemistry in 2002 from Buffalo State

College. She is a recipient of the Nanotechnology Fellowship Student Award (2003-2005) from the Center for Nanotechnology at the University of Washington and an MRS poster award (2005).

Chen can be reached by e-mail at chenj2@ u.washington.edu.

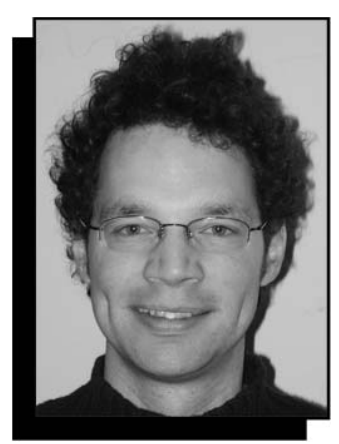

Aloyse Degiron

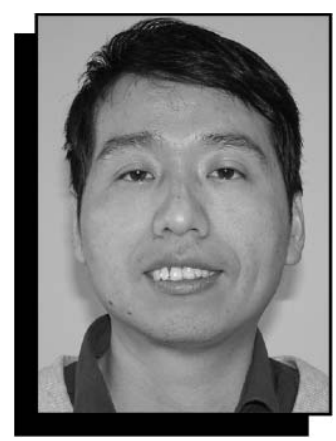

Zhi-Yuan Li

Aloyse Degiron is a postdoctoral researcher at Duke University in North Carolina, in the group of David Smith. He completed both his undergraduate and graduate studies at Louis Pasteur University in Strasbourg, France, receiving his PhD degree in physics in 2004. His current research is focused on a variety of phenomena associated with surfaceplasmon-enhanced transmission of subwavelength apertures.

Degiron can be reached at the Department of

Electrical and Computer Engineering, Duke

University, 130 Hudson Hall, Box 90291,

Durham, NC 27708, USA, and by e-mail at degiron@ee.duke.edu.

José Dintinger is a $\mathrm{PhD}$ student in physical chemistry at Louis Pasteur University in Strasbourg, France, where he also completed his undergraduate studies. His

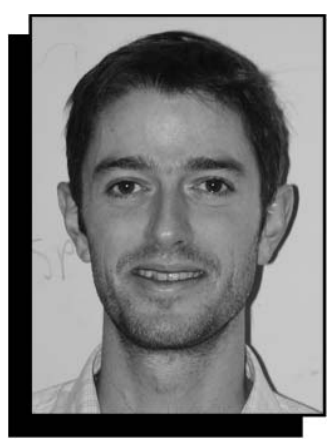

José Dintinger

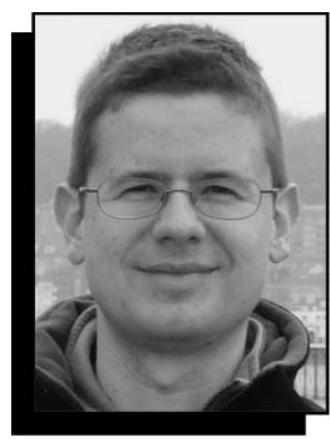

Stefan Maier

research interests include surface-plasmonmolecule interactions, strong coupling phenomena, and optical properties of nanostructured materials.

Dintinger can be reached at ISIS, Université Louis Pasteur, 8 allée Monge, 67000 Strasbourg, France, and by e-mail at dintinger@isis-ulp.org.

Jennifer A. Dionne is a doctoral candidate in the Applied Physics Department at the California Institute of Technology. She graduated from Washington University in St. Louis in 2003, receiving BS degrees in physics and systems science and engineering. Since joining the applied physics program at CalTech, her research has focused on understanding light-matter interactions on the nano-scale, emphasizing the analysis and design of plasmon waveguides for device and sensor applications. She is a National Science 


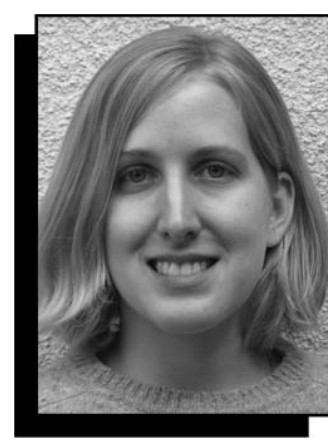

Jennifer A. Dionne

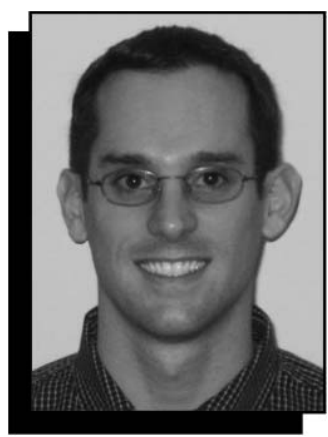

Adam D. McFarland

Foundation Graduate Research Fellow and a recipient of the National Defense Science and Engineering Graduate Fellowship.

Dionne can be reached at the California Institute of Technology, Watson Laboratories of Applied Physics, Mail Code 128-95, Pasadena, CA 91125, USA; tel. 626395-2380, fax 626-8449320, and e-mail jdionne@caltech.edu.

Thomas W. Ebbesen is a professor of chemistry at Louis Pasteur University in Strasbourg, France, and director of the Institute for Supramolecular Science and Engineering (ISIS). He received his BA degree from Oberlin College in Ohio (1976) and his PhD degree in physical chemistry from Pierre \& Marie Curie University in Paris (1980). He then spent an extended period in the United States and Japan, working, among other places, at the NEC Fundamental

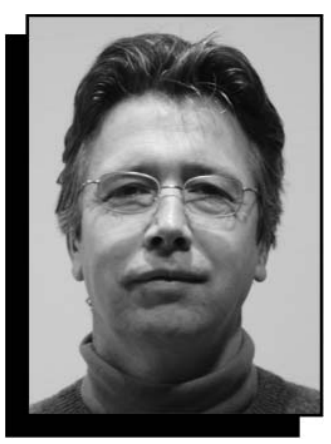

Thomas W. Ebbesen

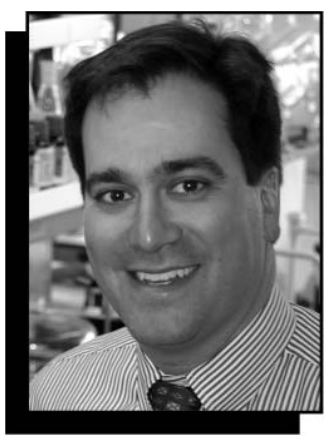

Chad A. Mirkin

Laboratory in Tsukuba and the NEC Research Institute in Princeton before returning to France in 1999. His research currently focuses on nanostructured materials with a particular emphasis on surface plasmon optics.

Ebbesen can be reached at ISIS, Université Louis Pasteur, 8 allée Monge, 67000 Strasbourg, France, and by e-mail at ebbesen@isis-ulp.org.

Anand Gole has been a postdoctoral research fellow in the Department of Chemistry and Biochemistry at the University of South Carolina since November 2003. $\mathrm{He}$ recieved his $\mathrm{PhD}$ degree from the National Chemical Laboratory in Pune, India, where he concentrated on problems related to protein-lipid and protein-gold colloid biocomposite materials. He also was a postdoctoral fellow at the Laboratoire de Physique des

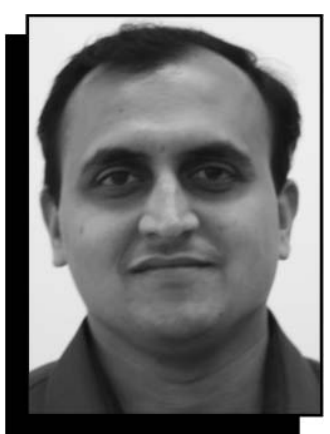

Anand Gole

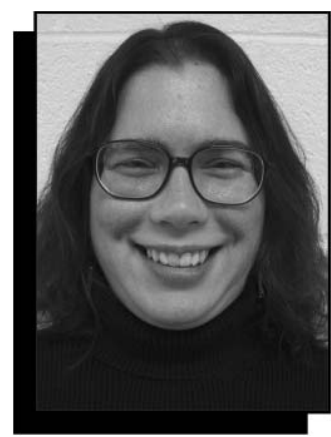

Catherine J. Murphy

Solides in Orsay, France, where he focused on polymer-surfactant and nanoparticle-lipid interactions at the air-water interface. His current research activities include the synthesis, characterization, and surface modification of onedimensional nanostructures and their subsequent assembly onto planar surfaces for possible sensing applications, nanoparticle cytotoxicity studies, and the formation of nano/bio assemblies.

Gole can be reached by e-mail at gole@mail. chem.sc.edu.

Amanda J. Haes is a National Research Council postdoctoral fellow at the U.S. Naval Research Laboratory in Washington, D.C. She received her BA degree in chemistry and physics from Wartburg College in 1999 and her MS and $\mathrm{PhD}$ degrees in chemistry from Northwestern University in 2001 and 2004, respec-

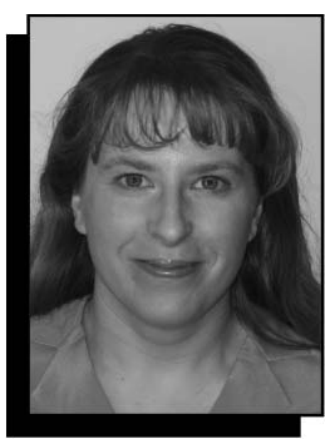

Amanda J. Haes

tively. Her research interests include nanoscience, plasmonics, surface-enhanced spectroscopy, and the integration of lithographic, microscopic, and spectroscopic techniques for biological and chemical sensor development.

She is a recipient of the Northwestern University Chemistry Department Award for Excellence in Graduate Research (2004), a Materials Research Society Graduate Student Gold Award (2003), an American Chemical Society Division of Analytical Chemistry and Dupont Research

Fellowship (2003), and a Kirkbright Bursary Award from the Association of British Spectroscopists (2004).

Haes can be reached by e-mail at ahaes@ccs. nrl.navy.mil.

Christy L. Haynes is currently a National Institutes of Health postdoctoral fellow in R. Mark Wightman's laboratory at the University of North Carolina, Chapel Hill, and will join the University of Minnesota in fall 2005 as an assistant professor of chemistry. She received her BA degree in chemistry from Macalester College in 1998, and her MS and PhD degrees in chemistry from Northwestern University in 1999 and 2003, respectively. Her research interests include nano-

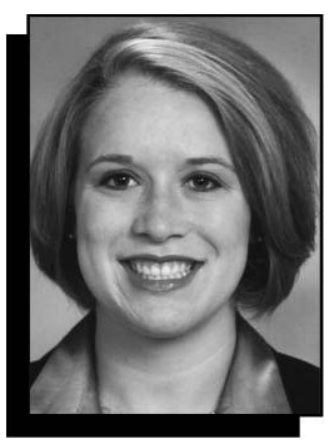

Christy L. Haynes

science, plasmonics, surface-enhanced spectroscopy, electrochemistry, neurochemistry, and the application of Raman spectroscopy to problems in neurobiology.

Haynes is the recipient of a Nobel Laureate Signature Award for Graduate Education from the American Chemical Society (2005), a Northwestern University Chemistry Department Award for Excellence in Graduate Research (2003), a Northwestern University Presidential Fellowship (2002), a Kirkbright Bursary Award from the Association of British Spectroscopists (2002), a Graduate Student Gold Award from the Materials Research Society (2002), and an American Chemical Society Analytical Chemistry Division Fellowship (2001).

Haynes can be reached by e-mail at haynes@chem.umn. edu.

Xingde $\mathbf{~ L i ~ i s ~ a n ~ a s s i s t a n t ~}$ professor of bioengineering at the University of Washington. He received his BS degree in applied nuclear physics from the University of Science and Technology of China (USTC) in 1990. He then earned his $\mathrm{PhD}$ degree in condensed-matter physics and biomedical optics from the University of Pennsylvania in 1998 (with Arjun G. Yodh 
and Britton Chance), at which point he joined the Research Laboratory of Electronics at MIT for postdoctoral training (with James G.

Fujimoto).

Li's research centers on low-coherence interferometry, high-resolution optical coherence tomography, light scattering, photon diffusion, lighttissue interactions, biological tissue absorption and scattering spectroscopy, optical molecular contrast agents, and optically based novel miniature biomedical devices. He has received a National Science Foundation CAREER Award and has authored or co-authored more than 30 peer-reviewed publications.

Li can be reached by e-mail at xingde@ u.washington.edu.

Zhi-Yuan Li is a professor of physics in the Institute of Physics at the Chinese Academy of Sciences in Beijing. He received his BS degree in optoelectronics from the University of Science and Technology of China (USTC) in 1994 and then worked as a graduate student on photonic crystals and near-field optics at the Institute of Physics, Chinese Academy of

Sciences. After obtaining his $\mathrm{PhD}$ degree in 1999,

he worked as a postdoctoral fellow at the Hong Kong University of Science and Technology, the University of Washington, and Ames National Laboratory. His research interests include photonic crystals, nanophotonics, near-field optics, and integrated optics.

$\mathrm{Li}$ is the co-author of more than 60 peerreviewed publications. He currently serves as a regular referee for several prominent physics and

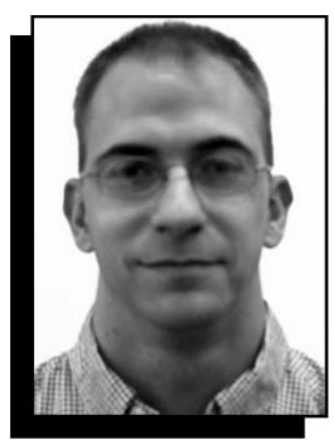

Christopher J.

Orendorff

optics journals, including Physical Review

Letters, Applied Physics

Letters, Physical Review B and $E$, and Optics

Letters.

Li can be reached by e-mail at lizy@aphy. iphy.ac.cn.

Stefan Maier is a lecturer in the Department of Physics at the University of Bath in the United Kingdom. His research interests include plasmonic nanostructures for energy guiding, confining, and biological sensing, and the interface between micro- and nano-optics. His work on plasmon waveguides has been recognized by numerous invitations to international conferences. He is also a frequent contributor to Spektrum der Wissenschaft.

Maier can be reached by e-mail at S.Maier@ bath.ac.uk.

\section{Adam D. McFarland} is a postdoctoral researcher in the Department of Materials Science and Engineering at

Northwestern University. He received his BS degree in chemistry from the University of Dayton in 1999 and his MS and PhD degrees in chemistry from Northwestern University in 2001 and 2004, respectively. His research interests focus on the development of analytical techniques and in-

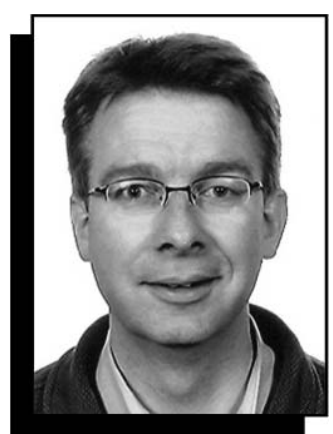

Albert Polman

strumentation for the characterization of nanoscale materials. His past research includes the utilization of single nanoparticles as chemical sensing platforms and the mechanistic study of surface-enhanced Raman spectroscopy. He is currently designing and constructing a lowtemperature scanning tunneling microscope for the study of nanoscale materials and molecular electronics.

McFarland can be reached by e-mail at mcfarlnd@chem. northwestern.edu.

Chad A. Mirkin is the George B. Rathmann Professor of Chemistry and a professor of medicine and materials science and engineering at

Northwestern University. $\mathrm{He}$ is also director of Northwestern's Institute for Nanotechnology. Mirkin is known for his invention and development of nanoparticlebased biodetection schemes, dip-pen nanolithography, and the weak-link approach to supramolecular coordination chemistry. He has authored more than 220 manuscripts and holds 50 patents. He is the founder of Nanosphere and NanoInk, two companies that are commercializing nanotechnology applications in the bio and semiconductor industries.

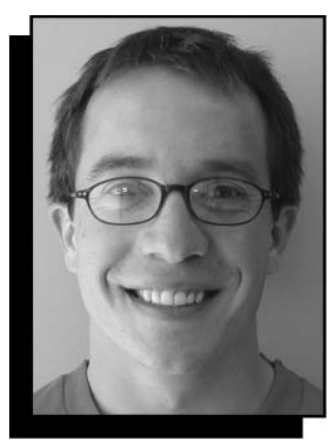

Nathaniel L. Rosi

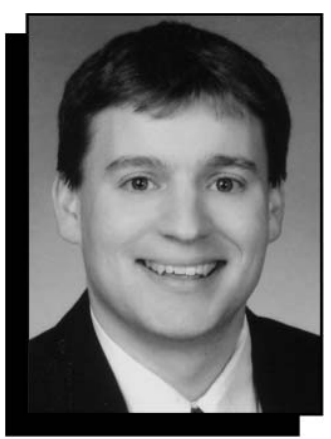

C. Shad Thaxton

He is a three-time winner of the National Inventors Hall of Fame Collegiate Inventors Award (1998, $2002,2004)$, and most recently, he was recognized with the $2004 \mathrm{NIH}$

Director's Pioneer Award.

Mirkin can be reached by e-mail at chadnano@ northwestern.edu.

Catherine J. Murphy is the Guy F. Lipscomb Professor of Chemistry at the University of South Carolina. She received BS degrees in chemistry and biochemistry in 1986 from the University of Illinois at UrbanaChampaign, and she received her $\mathrm{PhD}$

degree in inorganic chemistry from the University of Wisconsin in 1990. She was then an NSF and NIH postdoctoral fellow at the California Institute of Technology from 1990 to 1993. Her research interests include the synthesis, characterization, and optical applications of inorganic nanomaterials, coordination

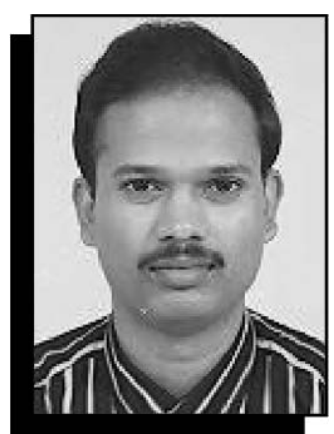

Tapan K. Sau

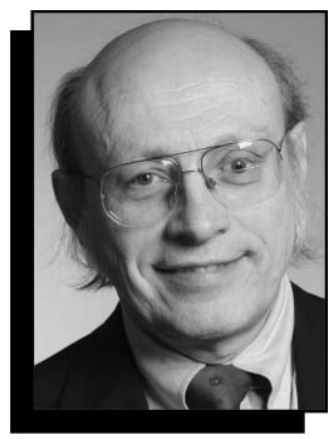

Richard P. Van Duyne

compounds for optical sensing, and optical probes of local DNA structure and dynamics. She has received a CAREER Award and a Special Creativity Award from the National Science Foundation and has been a Research Corporation Cottrell Scholar, an Alfred P. Sloan Foundation Research Fellow, and a Camille Dreyfus Teacher-Scholar. She is also an invited member of the Nanotechnology

Technical Advisory Group, which reports to the President's Council of Advisors on Science and Technology, and she serves on the editorial advisory boards for seven journals.

Murphy can be reached by e-mail at murphy@mail.chem. sc.edu.

Christopher J. Orendorff is a postdoctoral fellow in the Department of Chemistry and Biochemistry at the University of South Carolina. He earned his $\mathrm{PhD}$ degree in analytical 


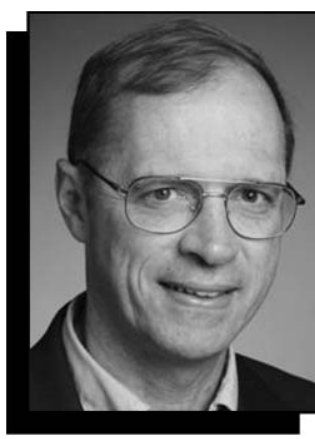

George C. Schatz

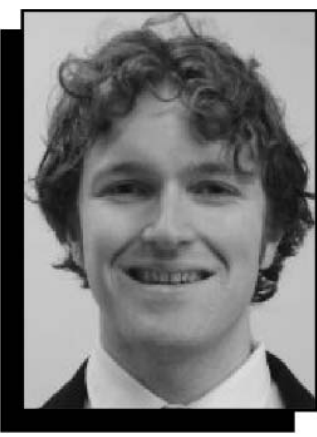

Benjamin Wiley

chemistry from the University of Arizona in 2003. His research focuses on using primarily metallic nanorods/wires for chemical and biological analysis and the rational manipulation of nanorods/wires in solution and on substrates.

Currently, he is working on patterning nanospheres and nanorods on solid supports using specific biological and chemical interactions, studying elastic lightscattering from nanoparticles of various shapes, studying the selfassembly and controlling the orientation of nanorods in different media, and developing the use of nanorods as sensors to determine physical stress and strain in polymers and biological tissues.

Orendorff can be reached by e-mail at orendorff@mail.chem.sc. edu.

Albert Polman is the head of the Center for Nanophotonics at the FOM Institute for Atomic

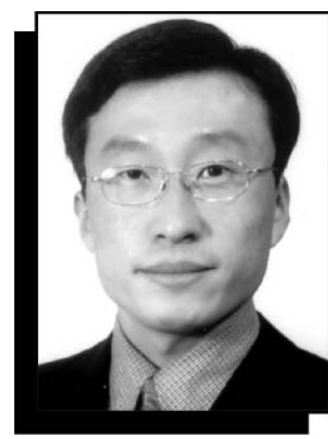

Yugang Sun

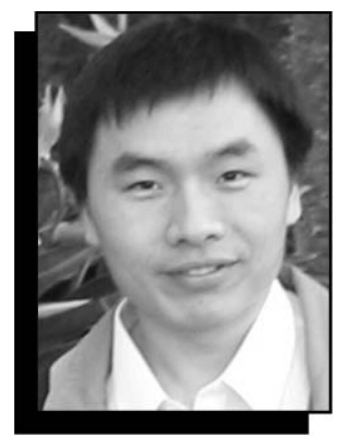

Shengli Zou

and Molecular Physics (AMOLF) in Amsterdam. His main research interest is nanophotonics, the study of optical interactions in materials made using nanoscale fabrication technologies. His group's research program includes the study of energy transfer in metallodielectric plasmonic structures, control of optical modes and spontaneous emission in photonic crystals and microresonators, and light emission from silicon using rare-earth ions or silicon nanostructures. He has published over 150 journal articles on optical phenomena in photonic nanomaterials.

Polman's Web site is www.erbium.nl; he can be reached by e-mail at polman@caltech.edu.

Nathaniel L. Rosi is working on postdoctoral studies as a member of Chad A. Mirkin's group at Northwestern University. He earned his BA degree at Grinnell College in 1999 and his $\mathrm{PhD}$ degree from the

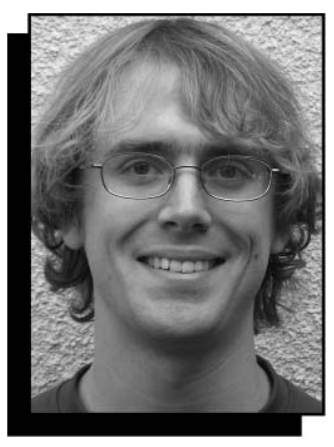

Luke Sweatlock

University of Michigan in 2003, where he studied the design, synthesis, and gas storage applications of metalorganic frameworks under the guidance of Omar M. Yaghi. His current research focuses on the rational assembly of DNA-modified nanostructures into larger-scale materials.

Rosi can be reached by e-mail at n-rosi@ northwestern.edu.

Tapan K. Sau is an assistant professor in the Department of Chemistry at Panjab University in Chandigarh, India, and is currently on a leave of absence working as a postdoctoral fellow with C.J. Murphy in the Department of Chemistry at the University of South Carolina. He obtained his PhD degree from the Department of Chemistry at the Indian Institute of Technology in Kharagpur.

Sau can be reached by e-mail at sau@mail. chem.sc.edu.

George C. Schatz is the Charles E. and Emma H. Morrison Professor of Chemistry at Northwestern University. He is a theoretician who studies the optical, structural, and energetic properties of nanomaterials, and he has contributed to theories of dynamical processes important in chemistry, including gas-phase and condensed-phase reactions, energy transfer processes, transport phenomena, and photochemistry. In the field of nanoscience, he has specialized in computational electrodynamic studies of noble metal nanoparticles, nanoholes, and other nanostructured materials. $\mathrm{He}$ has contributed to theories of DNA melting and nanoparticle aggregates, and he has studied the mechanical properties of nanotubes and thin films.

Schatz is a member of the American Academy of Arts and Sciences (2002), the International Academy of Quantum Molecular Sciences (2001), and is editor in chief of the Journal of Physical Chemistry.

Schatz can be reached by e-mail at schatz@ chem.northwestern.edu.

Yugang Sun is a research associate at the University of Illinois at UrbanaChampaign working with John Rogers. He received his $\mathrm{BS}$ and $\mathrm{PhD}$ degrees in chemistry from the University of Science and Technology of China (USTC) in 1996 and 2001, respectively. After graduating from USTC, he worked as a postdoctoral research associate with Younan $X i a$ at the University of Washington until the end of 2003. His research interests include the synthesis and characterization of nanostructures, micro- and nanofabrication, bioanalysis, and devices for photonics and electronics.

Sun can be reached by e-mail atygsun@ uiuc.edu.

Luke Sweatlock is a doctoral candidate in the Applied Physics Department at the California Institute of Technology. He obtained his BS degree in engineering physics at Cornell University and enrolled at Caltech following employment at Agere Systems' optoelectronics center. His research focus is on optical interactions in nanostructured metal composites and enabling the design and characterization of novel plasmonic devices.

Sweatlock can be reached at Caltech, MC 128-95, Pasadena, CA 91125 USA; tel. 626-3953826 and e-mail lukes@ caltech.edu.

C. Shad Thaxton is pursuing a career in academic urology at Northwestern University. He earned his BA degree at the University of Colorado in 1998 and his MD degree from Northwestern University's Feinberg School of Medicine in 2004. A Howard Hughes Medical Institute fellowship for medical students brought him to the Mirkin laboratory at Northwestern, where he worked on biomolecular detection using gold nanoparticle probes. His fellowship experience culminated with the reporting of the bio-barcode assay, for which he was honored with the National Inventors Hall of Fame Collegiate Inventors Competition graduate prize. He returned to the Mirkin lab to complete his $\mathrm{PhD}$ degree, where he continued to work on biomolecular detection and the use of gold nanoparticles for novel applications in the life sciences until beginning his current pursuit in academic urology.

Thaxton can be reached by e-mail at cthaxton003@ md.northwestern.edu.

Richard P. Van Duyne is the Charles E. and Emma H. Morrison Professor of Chemistry at Northwestern University. He is credited with the discovery of surface- 
enhanced Raman spectroscopy (SERS) and the invention of nanosphere lithography (NSL); he also developed ultrasensitive nanosensors based on localized surface plasmon resonance (LSPR) spectroscopy. His research interests include surfaceenhanced spectroscopy, nanofabrication, nanoparticle optics, combined scanning probe microscopy/Raman microscopy, Raman spectroscopy of massselected clusters, ultrahigh-vacuum surface science, structure and function of biomolecules on surfaces, and surface- enhanced spectroscopic methods for chemical and biological sensing.

Van Duyne is the recipient of several awards, including the Nobel Laureate Signature Award for Graduate Education from the American Chemical Society (2005), election to the American Academy of Arts and Sciences (2004), and the Earle K. Plyler Prize for Molecular Spectroscopy from the American Physical Society (2004).

Van Duyne can be reached by e-mail at vanduyne@chem. northwestern.edu.

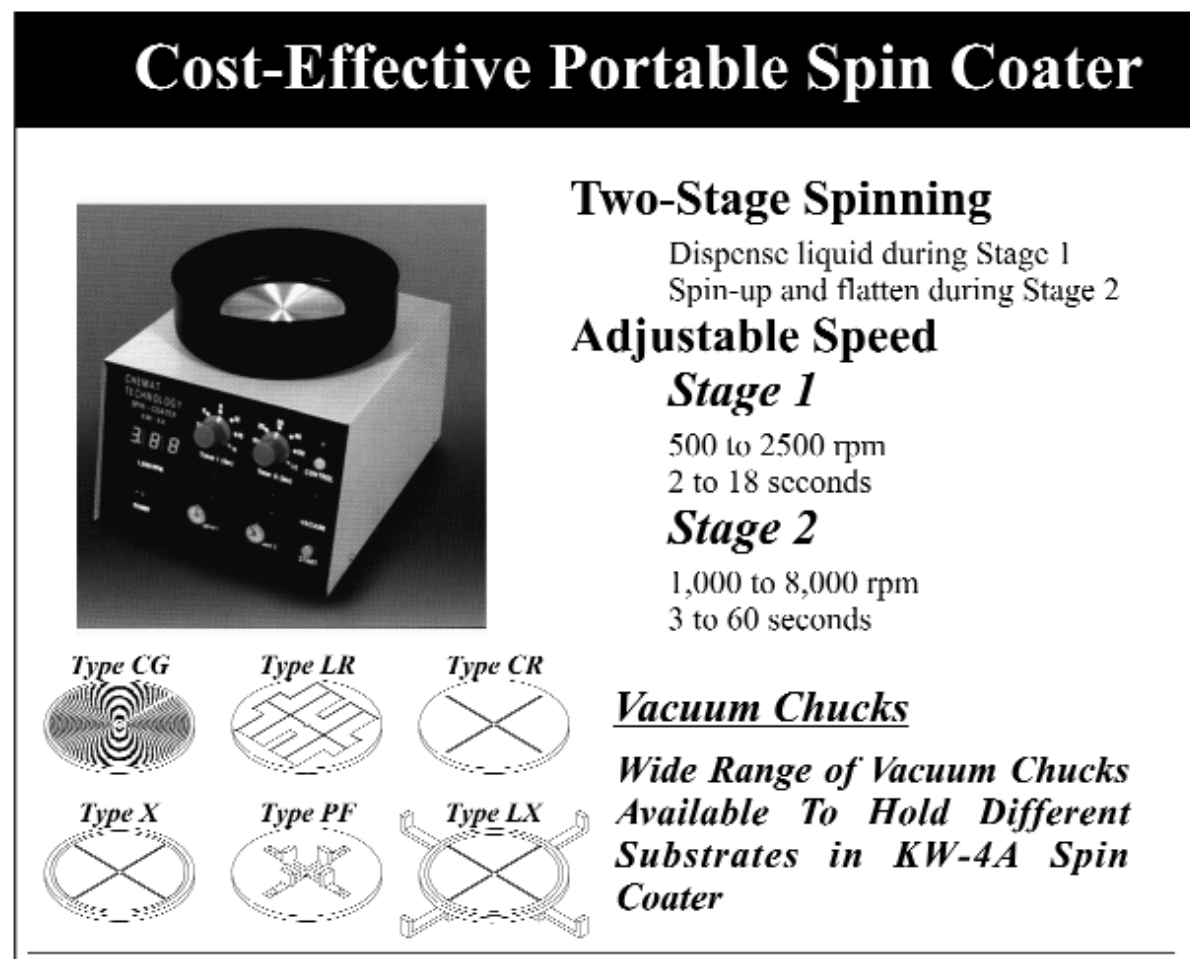

KW-4A SERIES PRODUCT LINE

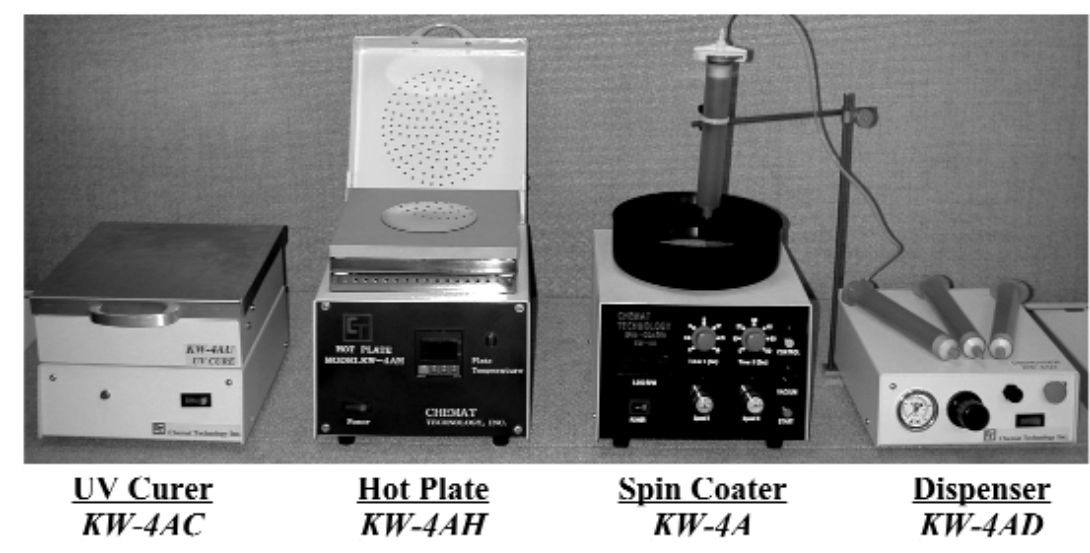

CHEMAT TECHNOLOGY, INC.

9036 Winnetka $\Lambda$ venue, Northridge, C $\Lambda$ 91324 1-800-475-3628, Fax: 818-727-9477 website: www.enlabproducts.com ; www.chemat.com email: marketing(a)chemat.com

For more information, see http://advertisers.mrs.org 